\title{
Towards non-invasive reflection measurement of water content in biotissue by means of terahertz time- domain spectroscopy
}

\author{
Mariia Borovkova ${ }^{1,2},{ }^{*}$ Mikhail Khodzitsky $^{1}$, Olga Cherkasova ${ }^{1,3}$, Alexey Popov ${ }^{1,2}$, Igor Meglinski ${ }^{1,2}$ \\ ${ }^{1}$ Department of Photonics and Optical Information Technology, ITMO University, St. Petersburg, Russia \\ ${ }^{2}$ Optoelectronics and Measurement Techniques Unit, University of Oulu, Oulu, Finland \\ ${ }^{3}$ Biophysics Laboratory, Institute of Laser Physics of the Russian Academy of Sciences, Novosibirsk, Russia
}

\begin{abstract}
We apply terahertz time-domain spectroscopy for the quantitative non-invasive assessment of water content in biological samples: tree leafs and pork muscles. The LandauLooyenga-Lifshitz-based model is used for the calculation of water concentration within the samples. The obtained results show that water content in biological samples can be measured utilizing terahertz waves in transmission and reflection modes.
\end{abstract}

Keywords-terahertz time-domain spectroscopy; water content in biotissue

\section{INTRODUCTION}

This study is devoted to the development and testing of the methods for non-invasive transmission and reflection measurements of water concentrations in biotissues by means of terahertz time-domain spectroscopy (THz TDS) [1]. Leaves and samples of pork muscles were tested.

\section{Methods AND MATERIALS}

The method to retrieve water concentrations is based on the effective medium theory and the extended Landau-LooyengaLifshitz model [2]. According to this model, a complex watercontaining sample can be considered as a compound consisting of several components, including water. It is possible to calculate the volume fractions of each of the component if the optical properties of the sample and each component are known. In this study, the tested samples were considered as a combination of two components: solid part of the sample and water. For this investigation, a series of experiments were performed utilizing THz TD spectrometer developed in-house operated in transmission and reflection modes.

Within this study, leaves of Carpinus caroliniana and pork muscles samples were tested. In the experimental protocol, several degrees of dryness of samples were artificially obtained using heating. The measurements of water concentration were performed at each of these stages using THz TDS. Simultaneously to the THz TDS measurements, the water concentrations were assessed gravimetrically for reference. In the last stage of the experiment, the weight value of the sample was considered as the weight of the dry component of the sample.

\section{RESUlTS AND CONCLUSIONS}

The values of water concentration obtained in the experiments at 5 stages of dryness are shown in Fig. 1 ((a) transmission, (b) - reflection modes). The results acquired by $\mathrm{THz}$ method are compared to the gravimetrical data.
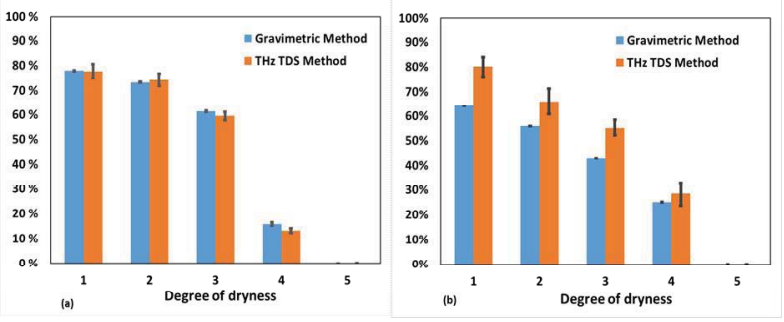

Fig. 1. Water concentration values retrieved by THz TDS and gravimetric methods at 5 stages of dryness for (a) a leaf of Carpinus caroliniana obtained in transmission mode, (b) pork muscle sample obtained in reflection mode.

The values of water concentration acquired by THz TDS in transmission [Fig. 1 (a)] correlate well with the gravimetrical data. The values of water concentration obtained by THz TDS in reflection [Fig. 1(b)] do not completely coincide with gravimetrical data. This is due to the fact that gravimetrical method can only assess average water concentration in the whole volume of a thick sample, whereas THz TDS measures water content in a spot of the superficial layer of the tissue.

We have demonstrated that THz TDS can be used to accurately determine water content in biological samples. This approach could provide valuable pathological insight, as it has the potential to distinguish healthy and injured tissues when visual assessment is problematic.

\section{REFERENCES}

[1] X. Zhang, Introduction To Thz Wave Photonics, Springer, 2014.

[2] A. I. Hernandez-Serrano, S. Corzo-Garcia, E. Garcia-Sanchez, M. Alfaro, and E. Castro-Camus, "Quality control of leather by terahertz time-domain spectroscopy," Applied Optics 53(33), 7872, 2014.

[3] N. Born, D. Behringer, S. Liepelt, S. Beyer, M. Schwerdtfeger, B. Ziegenhagen, and M. Koch, "Monitoring Plant Drought Stress Response Using Terahertz Time-Domain Spectroscopy," Plant Physiology 164(4), pp. 1571-1577, 2014 